\title{
The early days of letrozole
}

\author{
Ajay S. Bhatnagar
}

Received: 15 February 2007 / Accepted: 17 July 2007

(C) Springer Science+Business Media, LLC 2007

The year 2007 marks the 10th anniversary of the approval of letrozole (Femara ${ }^{\circledR}$ ) in the United States, while 2006 marked not only the 10-year milestone of letrozole's approval in Europe but also the 25th anniversary of Novartis's (then Ciba-Geigy [1970-1996]) dedication to the aromatase inhibitor (AI) program. It began with the introduction of the first-generation AI aminoglutethimide (Orimeten ${ }^{\mathbb{}}$ ) in 1981, progressed to the marketing of the steroidal AI formestane $\left(\right.$ Lentaron ${ }^{\mathbb{R}}$ ), development of the second-generation AI fadrozole (Afema ${ }^{\circledR}$ ), and finally led to letrozole, the most recent third-generation agent. This long track record in the development of AIs is unique in the pharmaceutical industry.

It is quite rare to be involved in the development of a drug that successfully completes the journey from discovery to approval and then advances care in a therapeutic field. The investigators involved in the early development of letrozole had such an opportunity.

At Ciba-Geigy (later Novartis), as in other pharmaceutical companies, thousands of compounds would be synthesized each year and, on average, only one or two would reach clinical development. In 1983-1984, the center of excellence for cancer research was transferred from the United States to Basel, Switzerland. As the newly appointed deputy head of the endocrine research department at Ciba-Geigy, I was assigned the AI project because of my previous experience and expertise in endocrine

With valuable contributions from Hilary Chaudri Ross, Henning T. Mouridsen, and Patrick Trunet.

A. S. Bhatnagar $(\bowtie)$

World Wide Services Group Ltd, Geispelgasse 13, CH-4132

Muttenz, Switzerland

e-mail: ajay.bhatnagar@wwsgroup.com projects with possible oncology indications. As part of the completion of the transfer of all oncology activities to Basel, the last batch of compounds synthesized by Dr. Robert Bowman arrived in Basel from the United States. Unknown to us, the compound that would become known as letrozole was among them. At this point in time, the second-generation AI fadrozole was undergoing clinical testing and was thus our lead compound, with all attention focused on it. The newly arrived compounds were stored in a freezer until an effective and robust in vivo assay was available. In 1986, when such an assay was developed in our labs, these last compounds were finally tested. The results of our early experiments showed that one compound appeared to be different from any of the others we had tested, in that it had a profound effect on the rat uterus. Considering that in those early days, compounds were screened at low $\mathrm{mg} / \mathrm{kg}$ doses and that aminoglutethimide was active at about $100 \mathrm{mg} / \mathrm{kg}$, we were very surprised to find that even at doses as low as a few hundred $\mu \mathrm{g} / \mathrm{kg}$, the new compound reduced the uterus to a very hair-like fine structure. It was at doses as low as $1 \mu \mathrm{g} / \mathrm{kg}$ that we saw dose-dependency in reducing uterine weight. This made our new compound infinitely more potent than any other AI previously tested.

Following this exciting preclinical discovery, and the subsequent preclinical profiling of letrozole as the most potent and selective AI tested, a brainstorming meeting was held to discuss the design of potential future clinical trials. Prof. Henning T. Mouridsen, one of the early investigators, speculated that this compound, now known as letrozole, showed great promise in revolutionizing breast cancer care. The letrozole program benefited tremendously in that it was not developed sequentially but rather dynamically, as a result of many discussions among all members of the letrozole team. Many protocol design meetings were 
held, and the input of outside physicians was carefully considered. Dr. Patrick Trunet, a Novartis clinician and the first International Project Team Leader for letrozole, was involved in the trial design, and he selected several external physicians he had previously worked with, including Prof. Mouridsen, his personal clinical experiment advisor. Other physicians involved in the clinical trial design included Drs. Richard Santen, James Ingle, Allan Lipton, Mitch Dowsett, and Ian Smith.

The enthusiasm over letrozole's potential grew even more following the completion of a successful in-house phase 1 study in healthy male volunteers, which used a letrozole solution instead of tablets in order to speed up the development process [1]. Due to the promising preclinical and phase 1 results, and once again to shorten development time, a novel phase 3 trial was designed using an innovative three-arm combined phase $2 \mathrm{~B} / 3 \mathrm{~A}$ design, which tested 2 doses of letrozole against each other and against a comparator. Mrs. Hilary Chaudri Ross, the team statistician, helped formulate the phase $2 / 3$ study design and kept us on course throughout the clinical development process. Mrs. Chaudri Ross was also intimately involved in other aspects of trial design, including decisions regarding end points and powering, and thus became the project statistician for all our AI trials and a core member of the project team. Her extensive involvement in the design of the clinical trial set a precedent for statisticians to be represented on all project teams, which is now standard at Novartis.

One of the important advantages of this novel trial design was that we were able to obtain results much faster than with the traditional phase 3 trial design. Our results showed that $2.5 \mathrm{mg}$ of letrozole was significantly more efficacious than the $0.5-\mathrm{mg}$ dose, and more importantly, that $2.5 \mathrm{mg}$ was significantly better than the comparator. The potency of letrozole was further demonstrated in a small experimental calibration trial in the first-line setting, where for the first time it appeared that letrozole might even be superior to tamoxifen. This finding allowed us to design a phase 3 trial, the Femara P025 study, which was powered to show the superiority rather than equivalence of letrozole over tamoxifen [2]. In addition, the P025 letrozole study had a unique crossover design. At an FDA advisory meeting, Novartis was complimented on having included a significant proportion of elderly women in trials and on involving three independent geographical areas (Europe, North America, and the rest of the world) in the P025 study, resulting in area-specific, reproducible results.

It is important to remember that letrozole was developed with one medical scientific concept in mind-that maximum estrogen suppression would lead to maximum tumor suppression. From the very beginning, we reasoned that by using the maximum tolerated dose of letrozole with maximum estrogen suppression, the stimulus for the hormonesensitive breast cancer can be most efficiently suppressed. Thus, efficacy was the most important aim, and the team believed that we were in the process of developing the most potent compound.

The team, led by Dr. Trunet, pushed from translational research to registration with extensive discussions on the concept of maximum estrogen suppression, new innovative clinical trial design, and long-term (10-year) strategy. It was one of the first projects within Ciba-Geigy/Novartis where Clinical and Research (and other line functions such as Drug Regulatory Affairs, Drug Metabolism and Pharmacokinetics, Toxicology, and Pharmaceuticals Development) worked together very closely. In addition, the same team was involved from research to market, which was one of the key success factors.

Subsequent endocrine profiling in humans showed that letrozole was an extremely powerful inhibitor of aromatase; and this, along with our conviction and belief in the compound, led to the fast-tracking of the letrozole clinical program within Novartis and to the subsequent launch of the drug. The first registration of letrozole for breast cancer was in France in mid 1996, and also in 1996, the drug was approved for the treatment of advanced breast cancer in Europe, with approval in the United States occurring 1 year later. It was first launched as first-line treatment for advanced breast cancer and for the neoadjuvant setting in the United Kingdom in 2001. Following the advanced breast cancer indication, letrozole received approvals in the extended adjuvant and most recently in the early adjuvant setting [3].

At the time letrozole was discovered, the general consensus in the medical community was that endocrine therapy with tamoxifen (Nolvadex ${ }^{\circledR}$ ) was the highest achievable standard of care for postmenopausal women with early breast cancer. The discovery and development of new agents such as letrozole was, therefore, met with early skepticism in the medical community; but the power of our results was convincing enough to accelerate the clinical trials needed to show that we were indeed dealing with a possibly new paradigm in the treatment of breast cancer.

All of the researchers involved in the development of letrozole were excited by the recognition that AIs represented an entirely new class of drugs with mechanisms of action different from those of tamoxifen and a different side-effect profile. The highly successful interaction and mutual respect between Novartis and external investigators was key to the rapid development of letrozole. Furthermore, the third-generation AI program established the concept of large, randomized trials. These large adjuvant studies sponsored by the pharmaceutical industry and guided by the scientific expertise of external investigators 
not only helped to unite the medical community but also greatly improved the quality of the data obtained in clinical studies.

Prior to the discovery of letrozole, many of us had a long history of conducting adjuvant endocrine trials. Interestingly, one of the first adjuvant endocrine studies, which compared tamoxifen with diethylstilbestrol (estrogen) versus control treatment, was run by Dr. Mouridsen [4]. In contrast with today's endocrine therapies, which involve complete estrogen inhibition, this study as well as other studies at the time supported the use of high-dose estrogen as standard endocrine therapy in postmenopausal women. Although estrogen therapy did show clinical efficacy, the treatment was toxic, with serious adverse events. Thus, paradoxically, the endocrine adjuvant treatment paradigm has evolved from estrogen administration to complete estrogen inhibition with the most potent class of drugs, the AIs.

It is unusual for a drug to be approved globally for multiple indications. Due to its efficacy and approval in all treatment settings, there is no doubt that letrozole will continue to contribute to advances in breast cancer care. After having looked back at the early days in the development of letrozole, it would be appropriate that we cast a glance into what the future might bring. One of the next exciting programs for AIs is combining them with signal transduction inhibitors. Since it is possible to block the estrogen receptor pathway almost completely with letrozole, the next step should be to target those growth pathways that may be up-regulated by complete blockade of the estrogen-dependent pathways. Thus, several studies investigating the efficacy of letrozole in combination with targeted therapies such as RAD001 [5], bevacizumab [6], or trastuzumab [7] are under way. Furthermore, emerging clinical data indicate that adjuvant letrozole therapy might be particularly effective in reducing the early risk of distant metastases, a well-recognized cause of breast cancer death $[8,9]$. The outstanding efficacy of letrozole in preventing distant recurrences suggests that letrozole is the optimal AI to give in the initial adjuvant setting, because early distant relapse prevention with letrozole should translate into improved overall survival. Future studies will provide additional insight into the full spectrum of the benefits of letrozole. What is clear from the data obtained over the last decade is that the full letrozole story is still unfolding.

\section{References}

1. Trunet PF, Mueller P, Bhatnagar AS, Dickes I, Monnet G, White G (1993) Open dose-finding study of a new potent and selective nonsteroidal aromatase inhibitor, CGS 20267, in healthy male subjects. J Clin Endocrinol Metab 77:319-323

2. Mouridsen H, Gershanovich M, Sun Y, Perez-Carrion R, Boni C, Monnier A, Apffelstaedt J, Smith R, Sleeboom HP, Janicke F, Pluzanska A, Dank M, Becquart D, Bapsy PP, Salminen E, Snyder R, Lassus M, Verbeek JA, Staffler B, Chaudri-Ross HA, Dugan M (2001) Superior efficacy of letrozole versus tamoxifen as first-line therapy for postmenopausal women with advanced breast cancer: results of a phase III study of the International Letrozole Breast Cancer Group. J Clin Oncol 19:2596-2606. Erratum in: J Clin Oncol 2001;19:3302

3. Femara package insert. East Hanover, NJ; Novartis Pharmaceuticals Corp., 2005

4. Palshof T, Mouridsen HT, Daehnfeldt JL (1980) Adjuvant endocrine therapy of breast cancer-a controlled clinical trial of oestrogen and anti-oestrogen: preliminary results of the Copenhagen breast cancer trials. Recent Results Cancer Res 71:185-189

5. Awada A, Cardoso F, Fontaine C, Dirix L, De Grève J, Sotiriou C, Steinseifer J, Wouters C, Tanaka C, Ressayre-Djaffer C, Piccart M (2004) A phase Ib study of the mTOR inhibitor RAD001 (everolimus) in combination with letrozole (Femara ${ }^{\mathbb{R}}$ ), investigating safety and pharmacokinetics in patients with advanced breast cancer stable or slowly progressing on letrozole. Breast Cancer Res Treat 88(Suppl 1):S234. Abstract 6043

6. Traina TA, Rugo H, Caravelli J, Yeh B, Panageas K, Bruckner J, Norton L, Park J, Hudis C, Dickler M (2006) Letrozole (L) with bevacizumab (B) is feasible in patients (pts) with hormone receptor-positive metastatic breast cancer (MBC). J Clin Oncol 24(18S): 133 s. Abstract 3050

7. Marcom PK, Isaacs C, Harris L, Wong ZW, Kommarreddy A, Novielli N, Mann G, Tao Y, Ellis MJ (2007) The combination of letrozole and trastuzumab as first or second-line biological therapy produces durable responses in a subset of HER2 positive and ER positive advanced breast cancers. Breast Cancer Res Treat 102:43-49

8. Coates AS, Keshaviah A, Thurlimann B, Mouridsen H, Mauriac L, Forbes JF, Paridaens R, Castiglione-Gertsch M, Gelber RD, Colleoni M, Lang I, Del Mastro L, Smith I, Chirgwin J, Nogaret JM, Pienkowski T, Wardley A, Jakobsen EH, Price KN, Goldhirsch A (2007) Five years of letrozole compared with tamoxifen as initial adjuvant therapy for postmenopausal women with endocrine-responsive early breast cancer: update of study BIG 1-98. J Clin Oncol 25:486-492

9. Mauriac L, Keshaviah A, Debled M, Mouridsen H, Forbes J, Thuerlimann B, Paridaens R, Gelber R, Castiglione-Gertsch M, Goldhirsch A (2006) Predictors of early recurrence in postmenopausal women with hormone receptor positive breast cancer in the BIG 1-98 trial. Eur J Cancer Suppl 4:111. Abstract 219 